\title{
Medical images classification for skin cancer using quantitative image features with optical coherence tomography
}

\author{
Wei Gao*, Valery P. Zakharov, Oleg O. Myakinin, Ivan A. Bratchenko, \\ Dmitry N. Artemyev and Dmitry V. Kornilin \\ Department of Laser and Biotechnical Systems \\ Samara State Aerospace University \\ *g.gaowei@gmail.com
}

Received 3 February 2015

Accepted 22 June 2015

Published 27 July 2015

\begin{abstract}
Optical coherence tomography (OCT) is employed in the diagnosis of skin cancer. Particularly, quantitative image features extracted from OCT images might be used as indicators to classify the skin tumors. In the present paper, we investigated intensity-based, texture-based and fractalbased features for automatically classifying the melanomas, basal cell carcinomas and pigment nevi. Generalized estimating equations were used to test for differences between the skin tumors. A modified $p$ value of $<0.001$ was considered statistically significant. Significant increase of mean and median of intensity and significant decrease of mean and median of absolute gradient were observed in basal cell carcinomas and pigment nevi as compared with melanomas. Significant decrease of contrast, entropy and fractal dimension was also observed in basal cell carcinomas and pigment nevi as compared with melanomas. Our results suggest that the selected quantitative image features of OCT images could provide useful information to differentiate basal cell carcinomas and pigment nevi from the melanomas. Further research is warranted to determine how this approach may be used to improve the classification of skin tumors.
\end{abstract}

Keywords: Optical coherence tomography; skin tumor; texture analysis; fractal analysis; differentiate box counting.

\section{Introduction}

Optical coherence tomography (OCT) is an optical imaging technique that has high dynamic range by the use of a broadband light source and heterodyne detection technique. ${ }^{1}$ The OCT technique has been used for the diagnosis of skin cancer because it can provide high resolution, cross sectional images in a noninvasive manner. By employing the OCT technique, the structural measurement of skin cancer can be obtained from OCT scans. The structural changes can be used to characterize the different types of skin cancer. The skin cancers include the

*Corresponding author.

This is an Open Access article published by World Scientific Publishing Company. It is distributed under the terms of the Creative Commons Attribution 3.0 (CC-BY) License. Further distribution of this work is permitted, provided the original work is properly cited. 
melanomas, basal cell carcinomas and pigment nevi. Particularly, it is clinically important to find the quantitative imaging biomarker of basal cell carcinomas and pigment nevi.

The quantification of the structural measurement with OCT has helped to classify the skin cancer. ${ }^{2}$ However, the direct information from OCT images is the reflectance (intensity). The structural measurement is obtained later from reflectance. Therefore, the intensity-based parameters might be used as the indicators to differentiate skin cancers. Moreover, in biology and medicine, the shapes of structures such as molecules, cells, tissues and organs play an important role in the diagnosis of diseased tissue., ${ }^{3,4}$ The utilization of texture analysis and fractal analysis could provide further information regarding the classification of skin cancers.

Our aim was to investigate the possibility of OCT to classify the skin cancers based on the intensity-based, texture-based and fractal-based features. OCT images were obtained from tissues with melanomas, basal cell carcinomas and pigment nevi. Intensity histogram and absolute gradient were calculated. The texture analysis was performed on OCT images. The fractal dimension was calculated by using the differentiate box counting method.

\section{Material and Methodology}

\subsection{OCT system and data collection}

The custom-built OCT system used in this study employs a broadband light source, delivering an output power of $20 \mathrm{~mW}$ at the central wavelength of $840 \mathrm{~nm}$ with a bandwidth of $25 \mathrm{~nm}$ (see Fig. 1). It has a resolution of $3.7 \mu \mathrm{m}$ in tissue that determines the imaging axial resolution of the system.

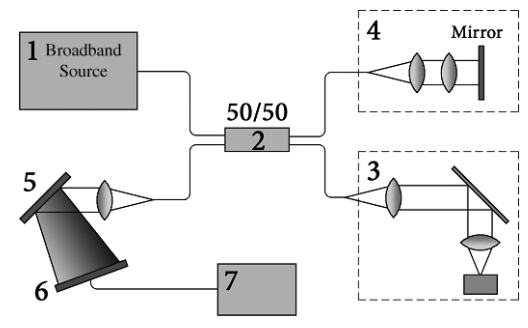

Fig. 1. Spectrometer-based spectral domain optical coherence tomography (SDOCT) system. 1 - broadband light source, 2 - 50/50 beamsplitter, 3 - sample arm, 4 - reference arm, 5 - spectrometer with grating, 6 - CCD camera, 7 - computer with IMAQ.
A Michelson interferometer splits the light in a 50/ 50 ratio along the reference arm and the sample arm. The spectrometer comprises of a diffraction grating (1200 grooves per $\mathrm{mm}$ ) and a CCD line scan camera $(29.3 \mathrm{kHz}$ line rate with 2048 pixel resolution). The signal is digitized by an image acquisition card (NI-IMAQ PCI-1428).

The Institutional Review Board of each institution (Samara State Aerospace University and Samara State Medical University) involved in the study approved the study protocol. The research adhered to the tenets set forth in the Declaration of Helsinki. Informed consent was obtained from each subject.

\subsection{Histopathological and OCT images}

Ex vivo experiments for skin tissues were performed. The skin tissue of pigment nevi was collected in the size of $11.5 \times 7 \mathrm{~cm}$ with $3.5 \times 2.5 \mathrm{~cm}$ light brown tumor from the right side of anterior abdominal wall. The skin tissue of the basal cell carcinoma was collected in the size of $4 \times 5 \mathrm{~cm}$ with $1.5 \mathrm{~cm}$ tumor diameter from the back. The skin tissue of the melanoma was collected in the size of $8 \times 3 \mathrm{~cm}$ with $1.5 \mathrm{~cm}$ in diameter mushroom-like tumor with rough black borders from the cheek.

Histopathological sections of studied skin samples were shown in Fig. 2. OCT images were shown in Fig. 3. Figure 2(a) is a microscope slide of skin tissue without signs of malignancy. The tissue which a basal layer is built with the structure of normal skin cells with a certain amount of pigment is characterized as pigmented nevi. Similar to the histopathological section, appropriate OCT image [see Fig. 3(a)] of studied nevi is defined by specific layered structure. In contrast to nevi sample basal cell carcinoma and melanoma tissue samples show signs of malignancy that help to distinguish them from normal tissue and nevi. Figure 2(b) represents histopathology section of basal cell carcinoma sample. One can clearly see the painted layers of tumor cells of round or elliptical shape. Basal cell carcinoma cells on the periphery of the tumor layer are typically in the form of a lance-like row. This formation of malignant cells defines changes in optical density of basal cell carcinoma and normal tissue so the basal cell carcinoma area has a darker color on the OCT image. The OCT picture of basal 


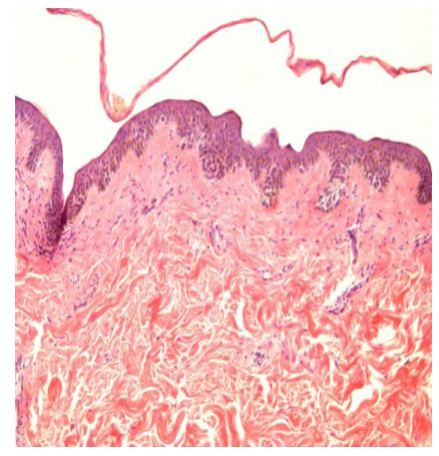

(a)

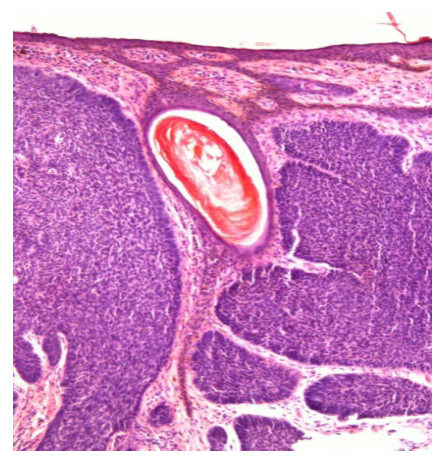

(b)

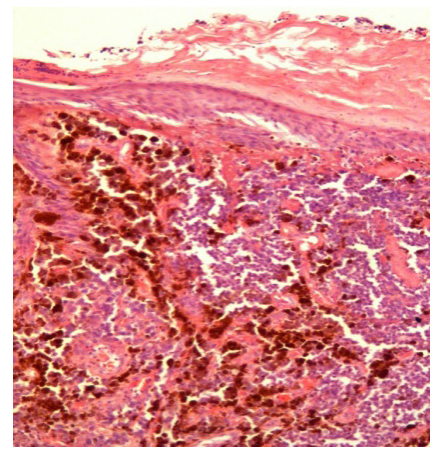

(c)

Fig. 2. Histopathological sections of studied skin samples (100 × magnification) (a) pigment nevi; (b) basal cell carcinoma; (c) melanoma.

cell carcinoma [see Fig. 3(b)] has easily observable tumor formations in the form of round or ellipsoidal "nests" in the right part of the image, while in the left part of the image normal structure of epidermis is visible (including the stratum corneum thin light layer and darker basal layer turning into the dermis). The histopathological section [see Fig. 2(c)] and OCT image [see Fig. 3(c)] of malignant melanoma represents a skin area of tumor and normal skin borders crossing location. On OCT, melanoma

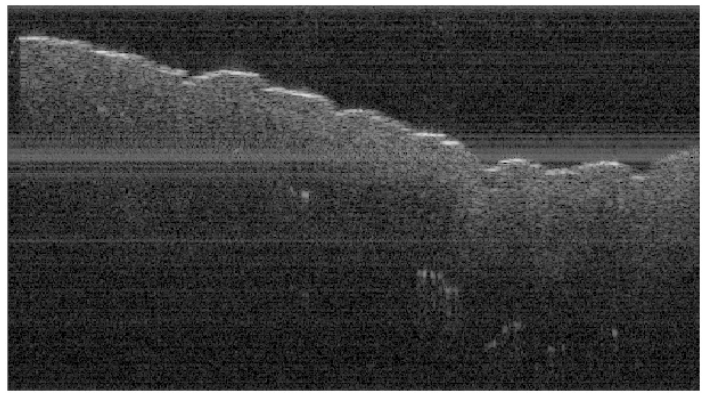

(a)

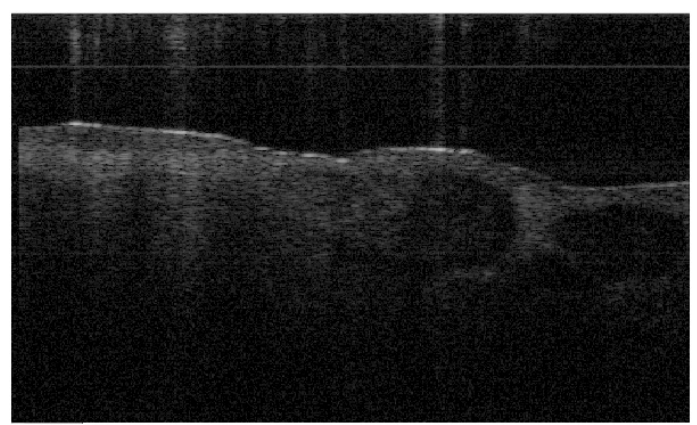

(b)

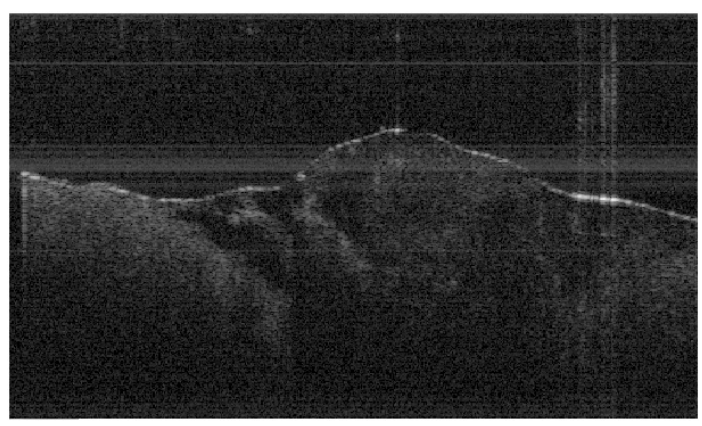

(c)

Fig. 3. OCT images of studied skin samples (a) pigment nevi; (b) basal cell carcinoma; (c) melanoma.

image normal epidermis (including the stratum corneum) is seen as a bright stripe on the tissue surface. Diffusely scattering cells containing melanin complexes and small undifferentiated cells without pigment are located under the epidermis layer. This determines the optical properties of the tumor. Both OCT and histopathology reveals destruction of specific layered structure of normal skin. Randomly located multiform objects of different optical density are visualized on OCT image instead of normal layered structure. This fact is due to the heterogeneity of tumor cells. Tumors include different cell types that can be seen from 


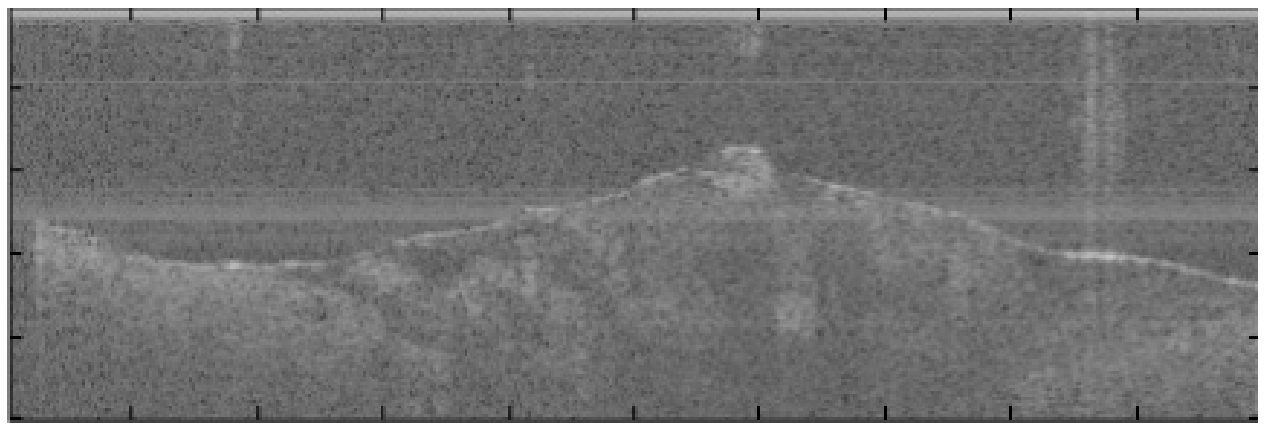

(a)

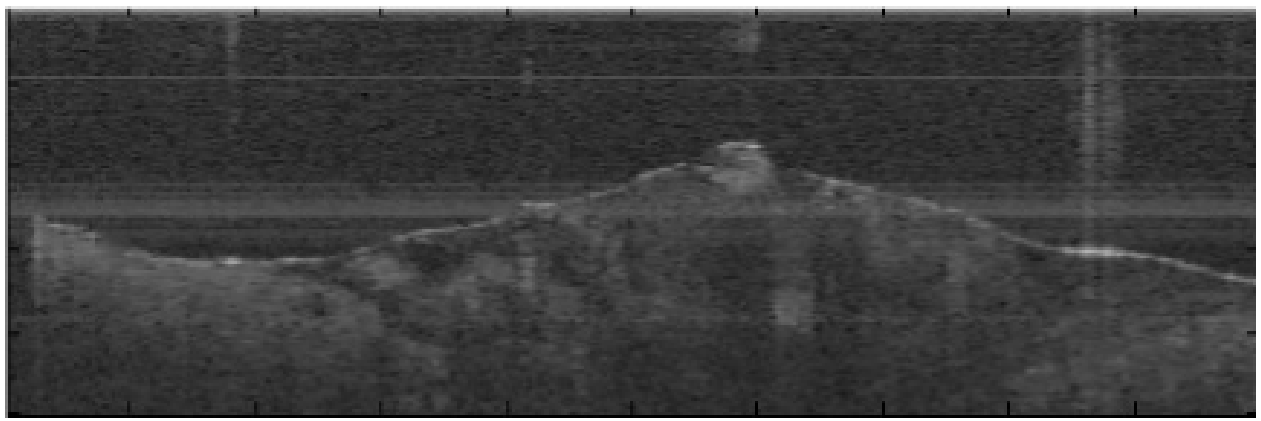

(b)

Fig. 4. Denoising results for a sample OCT scan. (a) Original OCT image. (b) Image denoised by using the interval type II fuzzy anisotropic diffusion filter.

histopathology section. Melanoma cells may have an excess amount of pigment or may include nonpigmented elements which appear on OCT image in the form of dark and bright areas, respectively.

\subsection{OCT image processing}

OCT signals were collected and exported from the OCT device in the form of 16-bit grayscale images. However, OCT images suffer from a particular type of noise called "speckle". The speckle is a common result of the limited spatial-frequency bandwidth of the interference signals in OCT. ${ }^{5}$ Because OCT imaging systems use coherent detection to generate images, speckle noise significantly degrades the OCT image contrast by creating a grainy appearance and by obscuring small, low intensity features; therefore, this makes it more difficult to extract the accurate image features from OCT images. To use image features to quantitatively classifying the skin tumors, the denoise of OCT images is required. We used the interval type II fuzzy anisotropic diffusion filter to remove the speckle noise from OCT images. ${ }^{6}$ The edge enhancement technique is also part of a novel preprocessing step that facilitates better feature extraction. Figure 4 demonstrates a sample OCT image before and after the speckle noise removal using the interval type II fuzzy anisotropic diffusion filter. Obviously, the utilization of the interval type II fuzzy anisotropic diffusion filter could not only effectively remove the speckle noise from OCT images but also enhance the sharp regions (e.g., edges).

\subsection{Quantitative image features}

In this section, quantitative image features were introduced. Those quantitative image features include the intensity-based, texture-based and fractalbased parameters (see Table 1). The methodologies for extracting quantitative image features from OCT images are also introduced.

\subsubsection{Intensity-based features}

The reflectance contained in OCT images not only represented the amplitude of OCT signals but also contained the information about the topographic 
Table 1. List of quantitative image features extracted from each OCT image.

\begin{tabular}{llll}
\hline $\begin{array}{l}\text { Intensity } \\
\text { histogram }\end{array}$ & $\begin{array}{l}\text { Absolute } \\
\text { gradient }\end{array}$ & Texture & $\begin{array}{c}\text { Fractal } \\
\text { analysis }\end{array}$ \\
\hline Mean & Mean & Contrast & Fractal dimension \\
Median & Median & Correlation & \\
Variance & Variance & Entropy & \\
Skewness & Skewness & Energy & \\
Kurtosis & Kurtosis & & \\
\hline
\end{tabular}

features and optical properties of the retinal tissue. In most biological tissues, the main sources of reflection are collagen fiber bundles. Dark areas on the image represent homogeneous material with low reflectivity, such as air or clear fluids. Thus, reflectivity has been used as the indicator to stage the diseased tissue in the analysis of OCT images. ${ }^{7}$

Among the intensity-based parameters, intensity histogram and absolute gradient play an important role in image analysis. The histogram is a graph showing the number of pixels in an image at each different intensity value found in the image. An image gradient is a directional change in the intensity in an image. Image gradient may be used to extract information such as edge or shape from images. The quantitative image features regarding intensity histogram and absolute gradient are utilized. Those features are mean, median, variance, skewness and kurtosis of intensity histogram and absolute gradient.

\subsubsection{Texture-based features}

Texture is a property that represents the surface and structure of an image. Generally speaking, texture can be defined as a regular repetition of an element or pattern on a surface. ${ }^{8}$ Textures are complex visual patterns composed of regions with sub-patterns with the characteristics of size, shape, shades, brightness and spatial arrangement. Moreover, a textured area in an image can be characterized by a nonuniform spatial distribution of gray levels or intensities. The variation in intensity characterizes a texture and reflects the physical variation in the underlying scene. By analyzing the spatial arrangement of color or intensities in an image or selected region of interest (ROI), the image's irregularities can be measured.

Accordingly, texture parameters such as, energy, entropy, correlation and contrast were extracted by using second-order statistical texture analysis. ${ }^{9}$ The spatial gray level co-occurrence matrices (SGLDMs) suggested by Haralick have been widely used to estimate the texture features related to secondorder statistical texture analysis. ${ }^{10}$ SGLDMs were obtained for ROIs based on the estimation of the second-order joint conditional probability density function $s_{\theta}(i, j \mid d)$. Each $s_{\theta}(i, j \mid d)$ denotes the probability of a pixel with a gray-level value " $i$ " being a " $d$ " pixel away from another pixel of gray-level value " $j$ " in the " $\theta$ " direction. Then, four texture parameters including energy, entropy, correlation and contrast were calculated from SGLDMs. Energy denotes the sum of the sum of the square of each value in a local neighborhood:

$$
\text { Energy }=\sum_{i=0}^{L-1} \sum_{j=0}^{L-1}\left[s_{\theta}(i, j \mid d)\right]^{2},
$$

where $L$ is the number of gray levels in the image.

Entropy denotes a measure of information content by measuring the randomness of the intensity distribution:

$$
\text { Entropy }=\sum_{i=0}^{L-1} \sum_{j=0}^{L-1} s_{\theta}(i, j \mid d) \log \left[s_{\theta}(i, j \mid d)\right] .
$$

Contrast denotes a measure of the local variations present in an image:

$$
\text { Contrast }=\sum_{i=0}^{L-1} \sum_{j=0}^{L-1}(i-j)^{2} s_{\theta}(i, j \mid d) .
$$

Correlation denotes a measure of image linearity. The value of correlation will be high if an image contains a considerable amount of linear structure:

\section{Correlation}

$$
=\frac{\sum_{i=0}^{L-1} \sum_{j=0}^{L-1}\left(i-\mu_{x}\right)\left(j-\mu_{y}\right) s_{\theta}(i, j \mid d)}{\sigma_{x} \sigma_{y}},
$$

where $\mu_{x}, \mu_{y}$ and $\sigma_{x}, \sigma_{y}$ denote the mean and standard deviations of the row and column sums of the gray level dependence matrices $s_{\theta}(i, j \mid d)$, respectively.

\subsubsection{Fractal-based feature}

The fractal dimension was first used to describe the self-similar pattern in the coastline of Britain by Mandelbrot in 1967. Mandelbrot found that the measured length of coastline changed as a different 


\section{W. Gao et al.}

size of measuring ruler was used. The fractal dimension was introduced and interpolated as a scale that was applied to the ruler used to measure the length of coastline. The scale can be regarded as a characterization that is used to describe the roughness of a surface such as the coastline. Due to this characterization, the fractal dimension was commonly used for the evaluation of the complexity of an object. Higher values indicate rougher surfaces. In the analysis of OCT images, fractal analysis has been used to investigate the structural change of biological tissue. For example, Fluearu et al. used the box counting method to calculate the fractal dimension to characterize porcine arterial tissue. ${ }^{11}$ Sullivan et al. utilized the box counting method to calculate the fractal dimension to classify the breast carcinoma. ${ }^{12}$ Gao applied the power spectrum method to perform the fractal analysis on the layered retinal tissue for diagnosing diabetic retinopathy. ${ }^{13}$ In those studies, fractal analysis was performed on each A-scan within each ROI. Therefore, only the irregularity or roughness along the A-scan was considered in the 1D fractal analysis, while the irregularity or roughness in all other directions was ignored. Thus, to extend the fractal analysis in all directions in two-dimensional OCT images, 2D fractal analysis is necessary.

To determine the fractal dimension for 2D image, several definitions of fractal dimension have been used. ${ }^{14}$ One simple and understandable methodology for calculating the fractal dimension is the differentiate box counting method. ${ }^{15}$ The methodology of differentiate box counting method can be described below. Consider an image of size $M \times M$ as a 3D spatial surface with $(x, y)$ denoting the $2 \mathrm{D}$ position on the image plane and the third coordinate $(z)$ denoting gray level (see Fig. 5). In the differentiate box counting method, the image plane is partitioned into nonoverlapping blocks of size $s \times s$, where $M / 2 \geq s \geq 2$ and $s$ is an integer. Then let an estimate of $r=s / M$. On each block, there is a column of boxes of size $s \times s \times s^{\prime}$, where $s^{\prime}$ is the height of each box. $G / s^{\prime}=M / s$ and $G$ is the total number of gray levels. Assign numbers $1,2, \ldots$ to the boxes as shown in Fig. 4. Let the minimum and maximum gray level in the $(i, j)$ th grid fall in the box number $k$ and $l$, respectively. The boxes covering this block are counted in the number as

$$
n_{r}(i, j)=l-k+1
$$

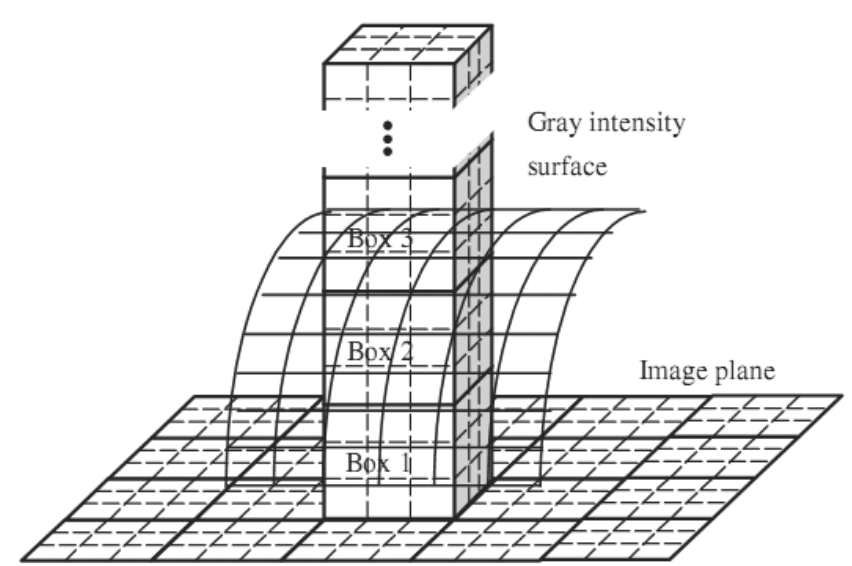

Fig. 5. Sketch of determination of the number of boxes by the DBC method. ${ }^{15}$

Taking contributions across all grids, we have

$$
N_{r}=\sum_{i, j} n_{r}(i, j),
$$

where $N_{r}$ is counted for different values of $r$. Then, the fractal dimension of $2 \mathrm{D}$ image can be estimated from the least square linear fit of $\log \left(N_{r}\right)$ versus $\log (1 / r)$.

\subsection{Statistical analysis}

Quantitative image features is reported after calculating from the OCT images of the melanomas, basal cell carcinomas and pigment nevi. The differences in quantitative image features between study groups were tested using an ANOVA followed by Newman-Keuls post-hoc analysis. Because of the number of comparisons, $p \leq 0.001$ was considered statistically significant. $0.001<p \leq 0.05$ was considered missed statistically significant. The ANOVA calculations and statistical analyses were performed using the software package Statistica version 8 (StatSoft Inc., Tulsa, Oklahoma).

\section{Results and Discussion}

The quantitative analysis of OCT data for diagnosing has been already proposed in fibrotic conditions where optical density has been used as the biomarker. ${ }^{16}$ While in this study, intensity-based, texture-based and fractal-based features were extracted from OCT images and then were used for the classification. Tables 2 and 3 showed the results of intensity-based parameters. Significant increases 
Table 2. Distribution statistics of intensity histogram (mean $\pm \mathrm{SD})$ by study groups.

\begin{tabular}{lccc}
\hline $\begin{array}{l}\text { Intensity } \\
\text { histogram }\end{array}$ & Melanomas & Nevi & Basal \\
\hline Mean & $6.294 \pm 1.919$ & $10.161 \pm 1.404^{\mathrm{a}}$ & $11.146 \pm 3.508^{\mathrm{b}}$ \\
Median & $6.237 \pm 2.141$ & $10.182 \pm 1.358^{\mathrm{a}}$ & $11.284 \pm 3.636^{\mathrm{b}}$ \\
Variance & $18.499 \pm 3.382$ & $10.275 \pm 2.309^{\mathrm{b}}$ & $20.399 \pm 3.343$ \\
Skewness & $0.044 \pm 0.393$ & $0.081 \pm 0.482$ & $-0.094 \pm 0.245$ \\
Kurtosis & $3.540 \pm 0.697$ & $4.285 \pm 1.772$ & $2.866 \pm 0.299$ \\
\hline
\end{tabular}

${ }^{\mathrm{a}} 0.001<p<0.05$

${ }^{\mathrm{b}} p<0.001$ (ANOVA followed by Newman-Keuls post hoc analysis) between melanomas and pigment nevi (see nevi column) and between melanomas and basal cell carcinoma (see basal column).

of mean and median of intensity distribution were observed when comparing basal cell carcinoma with melanomas (see Table 2). Specifically, the mean and median of intensity of basal cell carcinoma increased to $77.1 \%$ and $80.9 \%$ as compared to melanomas. When comparing pigment nevi with melanomas, the mean and median of intensity showed a missed statistical significant increase. And significant decreases of mean, median and variance of absolute gradient were observed when comparing basal cell carcinoma and pigment nevi with melanomas (see Table 3). As compared to melanomas, the mean, median and variance of absolute gradient in basal cell carcinoma decreased $16.6 \%, 16.6 \%$ and $28.1 \%$, respectively. As compared to melanomas, the mean, median and variance of absolute gradient of pigment nevi decreased to $23.8 \%, 25.4 \%$ and $34.8 \%$, respectively. Table 4 showed the result of texture analysis. Significant difference of contrast, entropy

Table 3. Distribution statistics of absolute gradient (mean $\pm \mathrm{SD})$ by study groups.

\begin{tabular}{lccc}
\hline $\begin{array}{l}\text { Absolute } \\
\text { gradient }\end{array}$ & Melanomas & Nevi & Basal \\
\hline Mean & $1.625 \pm 0.133$ & $1.238 \pm 0.080^{\mathrm{b}}$ & $1.355 \pm 0.063^{\mathrm{b}}$ \\
Median & $1.341 \pm 0.137$ & $1.000 \pm 0.079^{\mathrm{b}}$ & $1.118 \pm 0.061^{\mathrm{b}}$ \\
Variance & $1.386 \pm 0.171$ & $0.903 \pm 0.102^{\mathrm{b}}$ & $0.997 \pm 0.094^{\mathrm{b}}$ \\
Skewness & $1.476 \pm 0.196$ & $1.750 \pm 0.154^{\mathrm{a}}$ & $1.563 \pm 0.203$ \\
Kurtosis & $6.133 \pm 0.918$ & $7.969 \pm 1.223^{\mathrm{a}}$ & $7.080 \pm 1.637$ \\
\hline
\end{tabular}

${ }^{\mathrm{a}} 0.001<p<0.05$

${ }^{\mathrm{b}} p<0.001$ (ANOVA followed by Newman-Keuls post hoc analysis) between melanomas and pigment nevi (see nevi column) and between melanomas and basal cell carcinoma (see basal column).
Table 4. Distribution statistics of texture parameters (mean $\pm \mathrm{SD}$ ) by study groups.

\begin{tabular}{lccc}
\hline $\begin{array}{l}\text { Texture } \\
\text { analysis }\end{array}$ & Melanomas & Nevi & Basal \\
\hline Contrast & $2.666 \pm 1.647$ & $0.299 \pm 0.345^{\mathrm{b}}$ & $0.613 \pm 0.700^{\mathrm{b}}$ \\
Correlation & $0.665 \pm 0.048$ & $0.268 \pm 0.185^{\mathrm{b}}$ & $0.484 \pm 0.242^{\mathrm{a}}$ \\
Entropy & $0.914 \pm 0.567$ & $0.083 \pm 0.105^{\mathrm{b}}$ & $0.281 \pm 0.368^{\mathrm{b}}$ \\
Energy & $0.731 \pm 0.172$ & $0.977 \pm 0.028^{\mathrm{b}}$ & $0.928 \pm 0.095^{\mathrm{b}}$ \\
\hline
\end{tabular}

${ }^{\mathrm{a}} 0.001<p<0.05$

${ }^{\mathrm{b}} p<0.001$ (ANOVA followed by Newman-Keuls post hoc analysis) between melanomas and pigment nevi (see nevi column) and between melanomas and basal cell carcinoma (see basal column).

and energy were observed when comparing basal cell carcinoma with melanomas. Specifically, the contrast (entropy) of basal cell carcinoma decreased $77.0 \%$ (69.2\%) while the energy increased to $26.9 \%$ as compared to melanomas. When comparing pigment nevi with melanomas, the contrast, correlation and entropy showed the statistical significant decrease, while the energy showed the statistical significant increase. Table 5 showed the result of fractal analysis. Significant decrease of fractal dimension was observed when comparing basal cell carcinoma and pigment nevi with melanomas. The fractal dimension of basal cell carcinoma decreased to $6.1 \%$ as compared to melanomas. The fractal dimension of pigment nevi decreased to $6.7 \%$ as compared to melanomas.

Our results suggest that the lower reflectance was reflected from melanomas than others. This might be associated with pathological metabolic changes in the melanomas, where abundant hazardly distributed atypical melanocytes and pagetoid cells are present at all levels of the

Table 5. Distribution statistics of fractal dimension (mean $\pm \mathrm{SD}$ ) values by study groups.

\begin{tabular}{lccc}
\hline Fractal analysis & Melanomas & Nevi & Basal \\
\hline $\begin{array}{l}\text { Fractal } \\
\text { dimension }\end{array}$ & $2.290 \pm 0.099$ & $2.137 \pm 0.066^{\mathrm{b}}$ & $2.151 \pm 0.075^{\mathrm{b}}$ \\
\hline
\end{tabular}

${ }^{\mathrm{a}} 0.001<p<0.05$

${ }^{\mathrm{b}} p<0.001$ (ANOVA followed by Newman-Keuls post hoc analysis) between melanomas and pigment nevi (see nevi column) and between melanomas and basal cell carcinoma (see basal column). 
epidermis. ${ }^{17}$ Moreover, the dark areas in the deeper regions visible in the OCT images are possible necrotic or cystic regions. Our results showed the decreasing trend of contrast and entropy in basal cell carcinoma and pigment nevi as compared to melanomas. Contrast denotes the intensity difference; the high feature value represents image pixels with large intensity differences being in close proximity. Entropy gives higher values to regions that contain a wide variety of intensity distributions. The trend observed for the contrast and entropy may reflect the relatively large inhomogeneous intensities reflected from melanomas than basal cell carcinoma and pigment nevi. This fact may associate with the irregular and light-absorbing organs that were widely distributed in melanomas than in basal cell carcinoma and pigment nevi, which is coincident with the fact that energy showed increasing trend in basal cell carcinoma and pigment nevi. The reason is that it gives high feature values to image regions that have only a small number of intensity distribution patterns. This parameter is large in homogeneous scenes. Our results also indicated that the large fractal dimension was obtained in the melanomas than in basal cell carcinoma and pigment nevi. Fractal dimension was used as an indicator for the disorder or irregularity in the biological tissue. The larger value of fractal dimension in melanomas denotes the more disorder or irregularity inside this type of skin cancer. This might be associated with the fact that a denser network of unorganized vessels with chaotic branching in melanomas. This particular result also reflects the disorder inside the melanomas, which could be an indication that could be used as an additional indicator to enable the classification between melanomas and basal cell carcinoma.

There are some potential shortcomings of our study. The custom-built OCT technology has some limitations compared to the more advanced OCT technology. In addition, current OCT devices include different algorithms and methods for speckle noise removal. Therefore, data analysis is influenced by special assumptions and technological specifications that are in place for each individual OCT device. Another limitation is that only 1 of each lesion type was included in this study although 10 cross-sectional images were randomly selected for each lesion. However, more lesions rather than more scans of the same lesion would be beneficial to find the diagnostic parameters.

\section{Conclusion}

In summary, we have described a methodology to use the quantitative image features extracted from OCT images to classify the melanomas, basal cell carcinoma and pigment nevi in this paper. The early results presented have shown that the intensitybased, texture-based and fractal-based features could be used in the diagnosis of skin tumors. Particularly, the changes in intensity distribution and fractal dimension may reflect the pathological metabolic changes in melanomas. Future studies are needed to determine the accuracy, repeatability and full capability of this methodology with more OCT scans.

\section{References}

1. D. Huang, E. A. Swanson, C. P. Lin, J. S. Schuman, W. G. Stinson, W. Chang, M. R. Hee, T. Flotte, K. Gregory, C. A. Puliafito, J. G. Fujimoto, "Optical coherence tomography," Science 254, 1178-1181 (1991).

2. M. Mogensen, L. Thrane, T. M. Joergensen, P. E. Andersen, G. B. E. Jemec, "OCT imaging of skin cancer and other dermatological diseases," J. Biophoton. 2, 442-451 (2009).

3. P. G. H. Clarke, "Developmental cell death: morphological diversity and multiple mechanisms," Anat. Embryol. 181, 195-213 (1990).

4. C. D. M. Fletcher, Diagnostic Histopathology of Tumors, Churchill Livingstone (1995).

5. H. M. Salinas, D. Cabrera Fernandez, "Comparison of PDE-based nonlinear diffusion approaches for image enhancement and denoising in optical coherence tomography," IEEE Trans. Med. Imaging 26, 761-771 (2007).

6. P. Puvanathasan, K. Bizheva, "Interval type-II fuzzy anisotropic diffusion algorithm for speckle noise reduction in optical coherence tomography images," Opt. Express 17, 733-746 (2009).

7. W. Gao, E. Tatrai, V. Olvedy, B. Varga, L. Laurik, A. Somogyi, G. M. Somfai, D. C. DeBuc, "Investigation of changes in thickness and reflectivity from layered retinal structures of healthy and diabetic eyes with optical coherence tomography," J. Biomed. Sci. Eng. 4, 657-665 (2011).

8. G. N. Srinivasan, G. Shobha, "Statistical texture analysis," Proc. World Acad. Sci. Eng. Technol. 36, 1264-1269 (2008).

9. M. Sharma, M. Markou, S. Singh, "Evaluation of texture methods for image analysis," Proc. Seventh Australian and New Zealand Intelligent Information Systems Conference, Perth, 117-121 (2001). 
10. R. M. Haralick, "Statistical and structural approaches to texture," Proc. IEEE 67, 786-804 (1979).

11. C. Flueraru, D. P. Popescu, Y. Mao, S. Chang, M. G. Sowa, "Added soft tissue contrast using signal attenuation and the fractal dimension for optical coherence tomography images of porcine arterial tissue," Phys. Med. Biol. 55, 2317-2331 (2010).

12. A. C. Sullivan, J. P. Hunt, A. L. Oldenburg, "Fractal analysis for classification of breast carcinoma in optical coherence tomography," J. Biomed. Optics 16, 066010 (2011).

13. W. Gao, Improving the quantitative assessment of intraretinal features by determining both structural and optical properties of the retinal tissue with optical coherence tomography, Ph.D Thesis (2012).
14. M. Hasegawa, J. Liu, K. Okuda, M. Nunobiki, "Calculation of the fractal dimensions of machined surface profiles," Wear 192, 40-45 (1996).

15. N. Sarkar, B. B. Chaudhuri, "An efficient approach to estimate fractal dimension of textural images," Pattern Recogn. 25, 1035-1041 (1992).

16. G. Abignano, S. Z. Aydin, C. Castillo-Gallego, V. Liakouli, D. Woods, A. Meekings, R. J. Wakefield, D. G. McGonagle, P. Emery, F. D. Galdo, "Virtual skin biopsy by optical coherence tomography: The first quantitative imaging biomarker for scleroderma," Ann. Rheum. Dis. 72, 1845-1851 (2013).

17. B. R. Smoller, "Histologic criteria for diagnosing primary cutaneous malignant melanoma," Mod. Pathol. 19, S34-S40 (2006). 